\title{
Development of a dwell time calculation model for timetable planning
}

\author{
S. Buchmueller ${ }^{1}$, U. Weidmann ${ }^{1}$ \& A. Nash ${ }^{2}$ \\ ${ }^{1}$ Institute for Transport Planning and Systems (IVT), \\ Swiss Federal Institute of Technology (ETHZ), Switzerland \\ ${ }^{2}$ Vienna Transport Strategies (VTS), Vienna, Austria
}

\begin{abstract}
Accurately estimating station dwell time is critical for timetable planning. Its importance has increased as railways seek to improve timetable stability and network efficiency, while serving more passengers and different types of transport services. This research consisted of developing a station dwell time model in cooperation with the Swiss Federal Railways (SBB).

The proposed model estimates dwell times based on the input parameters: vehicle type (number, position, width and level of doorways), infrastructure (platform level) and demand (number and distribution of passengers). The research divides dwell time into five sub-processes: door-unblocking, opening doors, passenger boarding/alighting, closing doors and train dispatching. Each sub-process was evaluated separately to understand its influence on dwell time. The SBB's automatic passenger counting system was used to record the number of passengers boarding and alighting at each door and the beginning/ending time of each sub-process. During eight months over three million measurements were made on four different vehicle types operating on 20 different routes. These data were analyzed and used to develop the dwell time model.

This paper describes the research methodology, the structure of the dwell time model, the data collection system and presents a summary of results including statistical distribution and influence factors of sub-process times.
\end{abstract}

Keywords: timetable planning, dwell time, dwell process, boarding/alighting process, railway process times, S-Bahn train, regional train, automatic passenger counting system. 


\section{Introduction}

\subsection{Background}

Switzerland's suburban railway (S-Bahn) systems have become a victim of their own success. Passenger growth is increasing rapidly due to improved service quality (patronage on some lines has doubled in last 15 years). Unfortunately, the increased number of passengers has also increased critical service times such as station dwell time. The S-Bahn systems are operated in mixed traffic, in other words long-distance, suburban, regional and freight trains all share the same infrastructure. Finally, the Swiss railway network is operating close to capacity. This combination of factors has increased the risk of reducing timetable stability on the entire network.

One way of reducing this risk is to plan operations more precisely. An important part of this planning process is to accurately estimate station dwell time.

Therefore, the Swiss Federal Railways (SBB) asked the IVT to develop a universal dwell time calculation model.

\subsection{Research objectives}

The research objective was to develop a dwell time calculation model that allows planners to predict station dwell time (mean and spreading) by entering the following input parameters: vehicle type (number, position, width and level of doorways), station infrastructure (platform level) and demand (number and distribution of boarding and alighting passengers).

The output of the dwell time calculation model was defined as the time needed for a given number of passengers to board and alight the train at a specific stop. The model does not include the time needed to compensate for timetable margins (scheduled buffer times) or operational delays.

\subsection{Paper outline}

This paper is structured as follows: Section 2 describes the dwell process and its sub-processes. Section 3 describes the data collection system used to evaluate the dwell process. Section 4 presents results of the statistical evaluation of subprocess times and their influence factors. Section 5 presents conclusions including a description of the dwell time estimation tool and recommendations for further research.

\section{Analysis of the station dwell process}

A train's station dwell time is determined by the combination of the passenger boarding/alighting process, the door control system processes, and actions taken by the train driver and infrastructure operator (compare [1,2]). There are two main parts of the station dwell process: passenger service time and train dispatching time. In order to separate the influences of the different actors the station dwell process was divided into the 5 sub-processes shown in Table 1 . 
Table 1: $\quad$ Dwell time sub-processes.

\begin{tabular}{|l|l|l|l|}
\hline Sub-process & Location & Process Begins & Process Ends \\
\hline Door-unblocking (DU) & 1 Doorway & Train arrival & Begin door opening \\
\hline Door opening (DO) & 1 Doorway & Begin door opening & $\begin{array}{l}\text { First passenger } \\
\text { through the doorway }\end{array}$ \\
\hline $\begin{array}{l}\text { Boarding/alighting } \\
\text { (BA) }\end{array}$ & 1 Doorway & $\begin{array}{l}\text { First passenger } \\
\text { through the doorway }\end{array}$ & $\begin{array}{l}\text { Last passenger } \\
\text { through the doorway }\end{array}$ \\
\hline Door closing (DC) & 1 Doorway & $\begin{array}{l}\text { Last passenger } \\
\text { through the doorway }\end{array}$ & Door closed \\
\hline \hline Train dispatching (TD) & Whole Train & Last door closed & Train departure \\
\hline
\end{tabular}

As illustrated in Figure 1, since there are usually two or more doors per train, the first part of the dwell process - the passenger service time - can be understood as parallel sequences of the sub-processes DU, DO, BA and DC at each vehicle door (q.v. [1]). Due to unequal passenger distributions using vehicle doors and the stochastic spread of sub-process times, the last closing door determines the passenger service time. After the last door has been closed, the dwell process continues with the train dispatching sub-process.

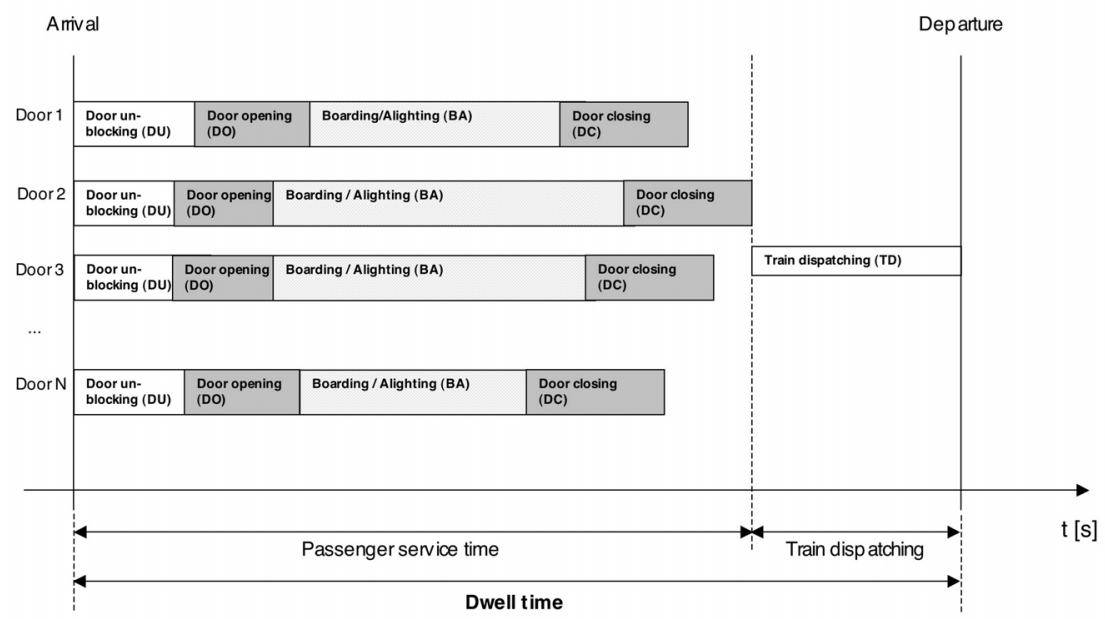

Figure 1: Dwell time sub-processes.

\section{Data collection: dwell time process measurement}

\subsection{Data collection requirements}

In order to estimate station dwell time, it is necessary to accurately measure the time needed to complete each sub-process in the dwell process and the number of passengers. These measurements must be very exact, many measurements are needed and they must be made in the complex operating environment of real-life 
dwell processes. Finally, all these data must be recorded for each vehicle door separately.

Given these stringent requirements, the SBB's automatic passenger counting system (AFZ) was modified and used for data collection.

\subsection{Automatic passenger counting system (AFZ)}

The SBB uses automatic passenger counting systems on most of its regional lines to gather passenger counts and to estimate ticket revenues. Approximately $30 \%$ of the fleet is equipped with AFZ (vehicles are rotated to cover all lines).

In normal operations the AFZ system registers the number of boarding and alighting passengers at each stop. The AFZ consists of chains of directionsensitive infrared-sensors above each doorway. These sensor chains are connected to a vehicle-wide network controlled by a central unit, which collects and stores the count data for the whole train over the course of the day. When the train arrives at its overnight storage yard these data are transmitted over wirelessLAN to a central database.

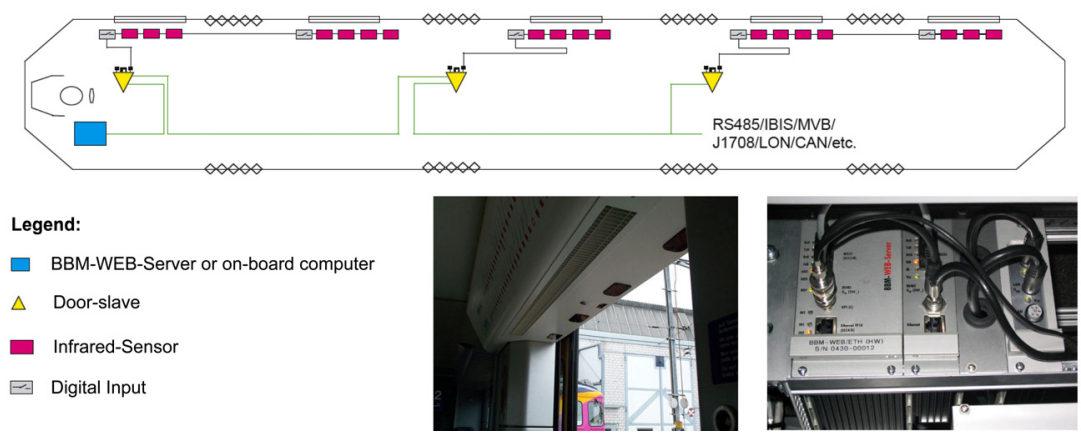

Figure 2: Automatic passenger counting system layout (above/left), infraredsensors over doorway (middle) and server unit (right) (Source [3]).

\subsection{Measurement parameters}

The AFZ system software had to be modified to record additional parameters (i.e. timestamps for each of the sub-processes) to obtain the data needed for the dwell time estimation model. Table 2 presents the parameters measured by the modified AFZ system used in this research.

Table 2: Dwell time parameters measured by the modified AFZ-system.

\begin{tabular}{|l|l|}
\hline Per train & For each door of the train \\
\hline Station ID & Number of boarding passengers \\
Train arrival (timestamp) & Number of alighting passengers \\
Train departure (timestamp) & Begin door opening (timestamp(s)) \\
& End door closing (timestamp(s)) \\
& First passenger through doorway (timestamp(s)) \\
& Last passenger through doorway (timestamp(s)) \\
\hline
\end{tabular}




\subsection{Data collection and validation}

The modified AFZ software was installed on 74 vehicles of 4 different types at the beginning of the data collection period. During the 8-month study period approximately 3.04 million dwell processes were measured and recorded. Table 3 summarizes the data collection by vehicle type.

Table 3: $\quad$ Dwell process measurements by vehicle type.

\begin{tabular}{|l|l|l|}
\hline Vehicle type & Type series / manufacturer & Measured dwell processes \\
\hline DTZ & RABe 514 / Siemens & 821 '488 \\
\hline FLIRT & RABe 523 / Stadler Rail & 1 '078'352 \\
\hline GTW 2/6 & RABe 526 / Stadler Rail & $655^{\prime} 574$ \\
\hline GTW 2/8 & RABe 526 / Stadler Rail & $489 ' 204$ \\
\hline
\end{tabular}

After collecting the data it was validated using a plausibility check in which the binary raw data were checked for error messages. Next, the files with valid raw data were transformed by software scripts and written into a database following a specifically defined data structure for use in the dwell time estimation model. Finally, the valid data were evaluated and analyzed; results of this analysis are presented in the following section.

\section{Station dwell sub-process time evaluation}

\subsection{Door unblocking sub-process (DU)}

The door unblocking (DU) sub-process time consists of the time period between the vehicle stopping in the station, as recorded by the train's central unit, and the beginning of door opening process, signalized by the local door control system and recorded by each door unit. The door opening process is considered to have begun when all the following conditions are met: (1) vehicle speed must be below a predefined value, (2) the train driver must have activated the pushbutton door opening controls at the vehicle doors and (3) door opening has been requested by the passengers.

New rail vehicles are often equipped with extensions that slide out at the doors to minimize the gap between the vehicle entrance and platform. The process of sliding-out the extension is an additional part of the DU sub-process: Due to safety restrictions this process cannot begin before the vehicle has stopped and it must be completed before the door begins to open.

Figure 3 presents the cumulative curves of door unblocking times for three different vehicle types under two scenarios (boarding + alighting passengers, and boarding passengers only). The following conclusions can be drawn from this data for the DU sub-process:

- Sliding extensions (present on vehicle types DTZ and FLIRT) cause median DU sub-process time to increase by between 2.9 to 3.5 seconds compared to vehicles with conventional entrances (vehicle type GTW). 
- Vehicles without sliding extensions are allowed to start door opening shortly before the train stops. Therefore these vehicle types can have negative DU sub-process times.

- Dwell processes with both boarding and alighting passengers have shorter median DU sub-process times ( 0.6 to 1.2 seconds) than dwell processes where passengers are only boarding. This is because alighting passengers may request door opening prior to train arrival in the station, but boarding passengers need time to reach the push-button door controls after train arrival in the station.

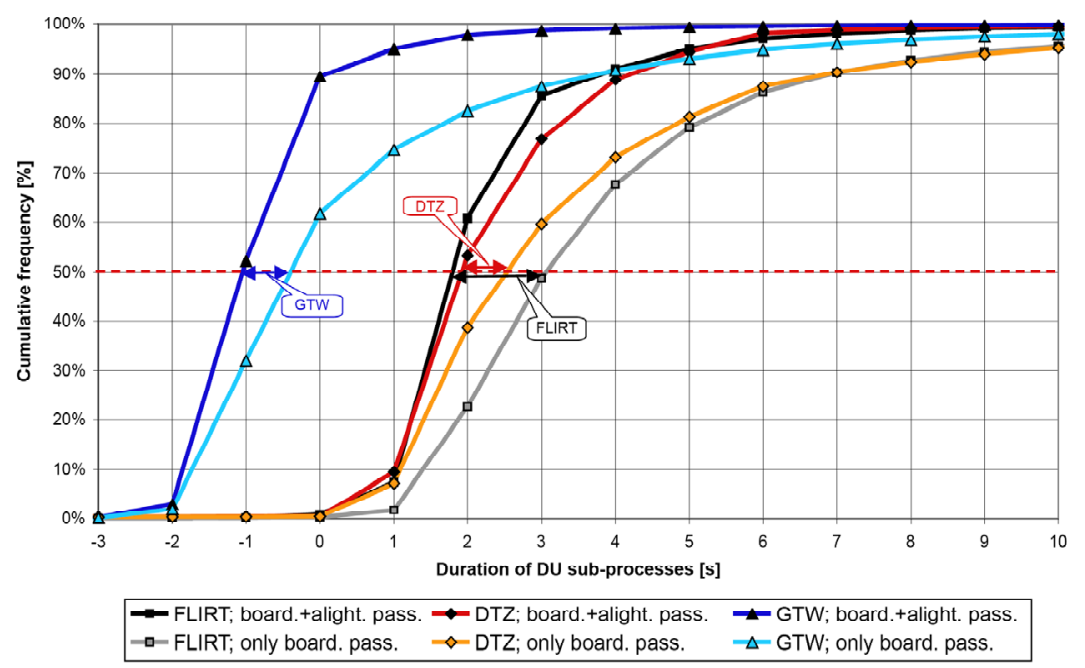

Figure 3: $\quad$ Cumulative frequency distribution of DU sub-process times.

\subsection{Door opening sub-process (DO)}

The door opening (DO) sub-process is the time from the start of door opening until the first passenger passes through the doorway.

Figure 4 presents the cumulative curves of door opening times for the three vehicle types and two scenarios.

The following conclusions can be drawn about DO sub-process times:

- The DO sub-process times are essentially determined by the door width and control system. The difference in DO sub-process time between the vehicle types evaluated in this study is less than 1 second (the vehicle door widths were between $1.3 \mathrm{~m}$ and $1.4 \mathrm{~m}$ ).

- Dwell processes with both boarding and alighting passengers have shorter median DO sub-process-times (by up to 0.8 seconds) compared to those with only boarding passengers. The main reasons are that alighting passengers are standing closer to the doorway than boarding passengers after they pushed the door-open buttons and that the infrared sensor are located just inside the vehicle doorway. 


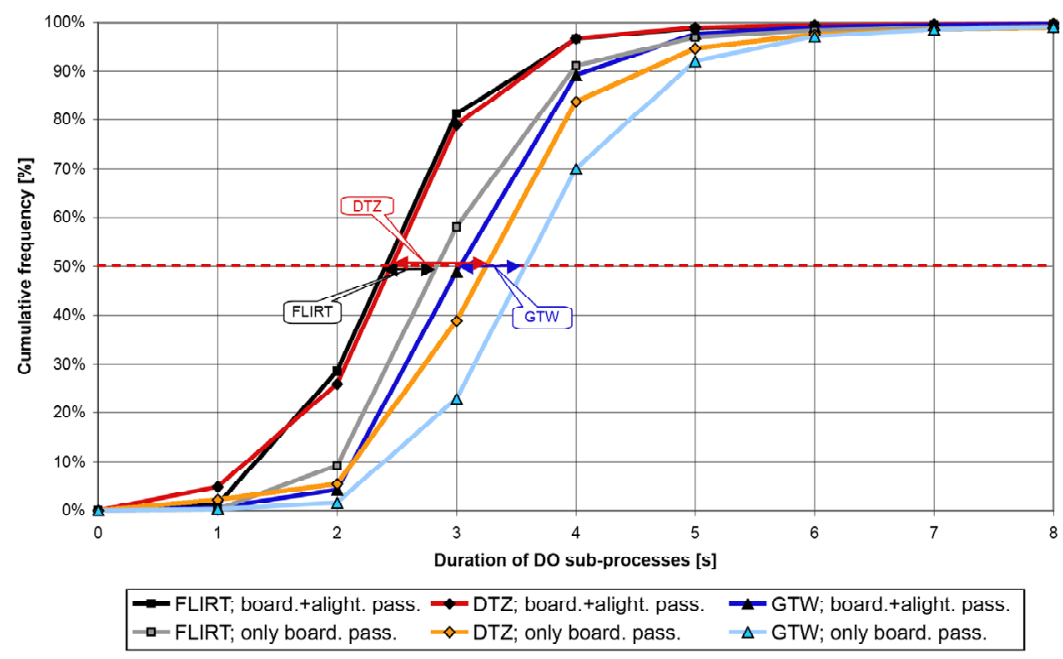

Figure 4: Cumulative frequency distribution of DO sub-process times.

\subsection{Boarding/alighting sub-process (BA)}

The boarding/alighting (BA) sub-process is the time period of passenger flows at the doorway. In order to obtain comparable values the BA sub-process was evaluated based on mean passenger flow rates (the ratio between the number of passengers and the BA sub-process time) calculated using the measured data.

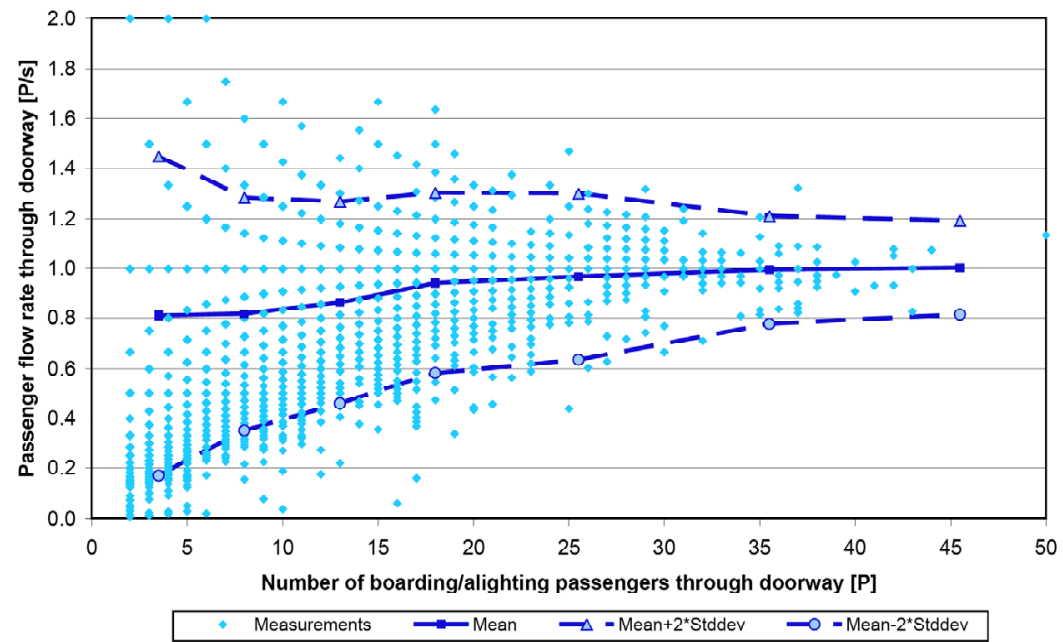

Figure 5: Passenger flow rates for FLIRT vehicles with vehicle occupancy of less than 90\%: measured flow rate (points), calculated mean (straight line) and mean $+/-2 *$ standard deviations (dashed lines). 
Figure 5 presents the mean passenger flow rates through the doorway compared to the number of boarding/alighting passengers for the FLIRT vehicles (which have level boarding). In Figure 5, each point represents one boarding/alighting process at a vehicle door. The average flow rate values and the standard deviations shown in Figure 5 were calculated based on these measurements and are displayed as a function of the number of passengers boarding/alighting.

The following conclusions can be drawn from the passenger flow evaluation:

- The evaluation confirmed results of earlier research (e.g. Weidmann [1]) that passenger flow at vehicle doors can be considered a normally distributed variable.

- The mean passenger flow rate through the doorway increases slightly with the number of boarding/alighting passengers per door.

- The standard deviation of the passenger flow rate decreases as the number of passengers boarding/alighting at each door increases.

- A further data evaluation showed that the passenger flow rate decreases continuously with occupancy once vehicle occupancy reaches $60 \%$ of seats occupied. This reduction is caused by the increasing number of conflicts between standing and moving passengers inside the vehicle and on the platform (Nash et al. [4], Lee et al. [5]).

\subsection{Door closing sub-process (DC)}

The door closing (DC) sub-process begins with the passage of the last passenger through the doorway. After a predefined vehicle-specific time period with no passengers boarding or alighting ("door remains open time"), the door begins closing. The sub-process ends with the door-closed-signal generated by the door control system. Note that this sub-process does not include retracting the sliding extension, at this point in time the sliding extensions are still in their extended positions.

Figure 6 presents cumulative curves of door closing sub-process time.

The following conclusions can be drawn from the door closing evaluation:

- The large difference in DC sub-process times between vehicle types is mainly the result of the different predefined "door remains open time" for different vehicles. This variation shows that the door closing process should be more carefully analyzed and adjusted to passenger behaviour.

- Dwell processes with boarding and alighting passengers have shorter median DC sub-process times (up 1.2 seconds) compared to those with only alighting persons. This can be explained by the relative positions of the AFZ counting sensor and the door closing control sensor and the walking direction of the last passenger.

\subsection{Train dispatching sub-process (TD)}

The train dispatching (TD) sub-process considers the whole train and consists of the time period between the moment when the last train door has closed and the train departure. The regional trains observed in this study were operated without 


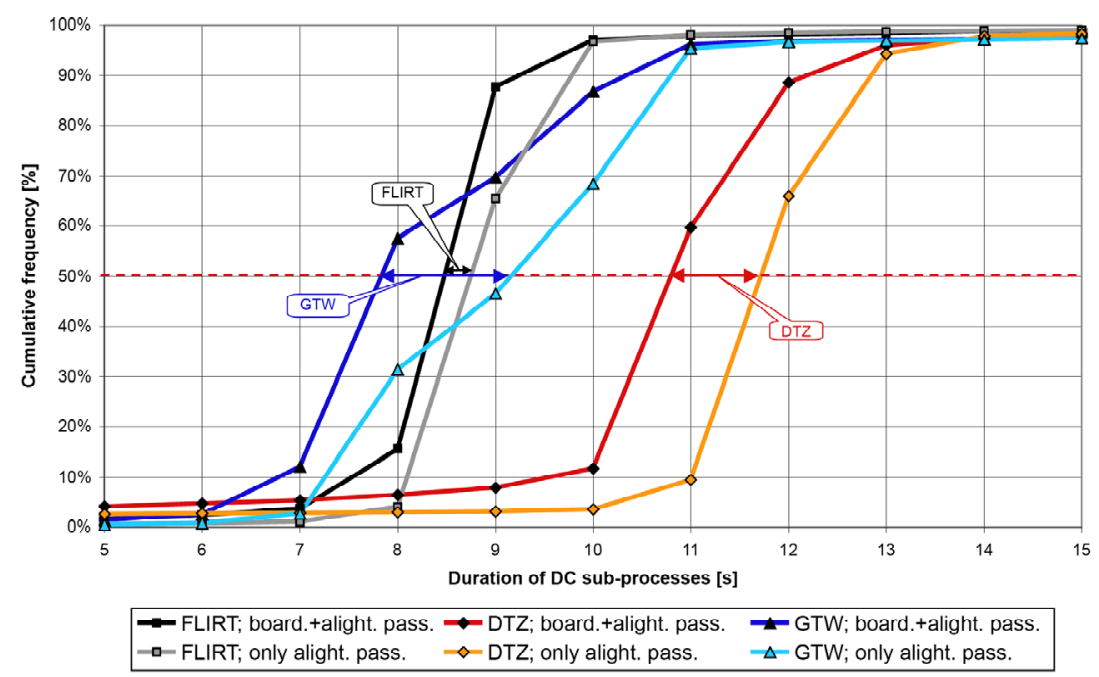

Figure 6: Cumulative frequency distribution of DC sub-process times.

conductors and therefore used an abbreviated train dispatching process executed by the train driver.

The TD sub-process duration is determined by the following factors (1) the process times for: door-blocking, retracting the sliding steps (if necessary) and starting-up the traction motors, (2) the waiting time for the scheduled departure time (if any), and (3) the delay caused by operational conflicts (if any).

Since these factors are highly dependant on the specific station, the spreading of TD sub-process times also depends on the station. Therefore, the dwell time calculation model was developed for stations where dispatching time is not significantly influenced by operational considerations. Operational considerations can be added to the model as required for a specific station.

\section{Conclusions and further research}

This research project has shown that passenger train station dwell time is determined by several different sub-processes taking place at each of the vehicle doors on a train. The project's data collection system made it possible to accurately measure a very large number of dwell sub-process times and additional parameters at all vehicle doors on a train. These measurement data made it possible to quantify and precisely model the sub-process times with respect to the relevant influence factors.

These data were used to develop a user-friendly dwell time calculation tool. It allows timetable planners to more accurately estimate station dwell time and use this information to develop more precise schedules. 
While the research advances understanding of the station dwell time process, it also points up the need for further research, specifically:

- Correlation between passenger flow rate and parameters including: (1) doorway width, (2) level differences between platform/train entrance, (3) vehicle occupancy, and (4) vehicle interior layout.

- The relationship between the distribution of passengers using specific vehicle doors and the following parameters: (1) the distances between vehicle doors, and (2) the location of $1^{\text {st }}$ and $2^{\text {nd }}$ class compartments.

- The relationship between the distribution of boarding passengers on the platform and the station layout (number and location of platform access points).

\section{Acknowledgements}

This research project was completed with support from the SBB. The authors appreciate the assistance and cooperation provided by the SBB project team without which it would have been impossible to achieve the project results.

\section{References}

[1] Weidmann, U., Grundlagen zur Berechnung der Fahrgastwechselzeit, Institute Report No. 106, Institute for Transport Planning and Systems, Swiss Federal Institute of Technology Zurich, Zurich, 1995

[2] Heinz W., Passenger service times on trains - theory, measurements, and models, Licentiate Thesis, Royal Institute of Technology, Stockholm, 2003

[3] Dilax Intelcom, Passenger Counting Systems, Functional principle, http:/www.dilax.com/en/index.htm?pages/pages/produkte/gesamt.htm

[4] Nash, A., Weidmann, U., Bollinger, S., Luethi, M. \& Buchmueller, S., Increasing Schedule Reliability on Zurich's S-Bahn Through Computer Analysis and Simulation, Transportation Research Record \#1955, Transportation Research Board, Washington D.C., pp. 17-25, 2007

[5] Lee, Y., Daamen, W. \& Wiggenraad, P., Boarding and alighting behavior of public transport passengers. In Proceedings of the $86^{\text {th }}$ Annual Meeting. CDROM. Transportation Research Board, Washington, D.C., pp. 1-14, 2007 